\title{
Recherche d'un programme de développement optimal de l'ensemble des réseaux de distribution d'eau potable en Région parisienne
}

\author{
par M. Dargent \\ Chef de la Division Ressources de l'Agence Financière \\ de Bassin Seine-Normandie
}

\author{
ef P. Bansard \\ Chef de Projet de la \\ SOGREAH
}

\section{Préambule}

L'alimentation en eau de la Région Parisienne est profondément marquée par l'évolution rapide de la démographie dans une banlieue largement étendue autour de la capitale, où un grand nombre de communes périphériques étaient jusqu'ici de type essentiellement rural.

Elle est caractérisée par l'existence de très nombreux réseaux indépendants, conçus avec un certain particularisme, et exploités, soit par des Régies communales ou syndicales, soit par des Sociétés privées spécialisées qui étendent progressivement leur zone d'activité. Le développement de l'urbanisation tend donc de plus en plus à rendre mitoyens les réseaux les plus importants qui n'ont cependant pas, entre eux, les liaisons qui permettraient à la fois une plus grande sécurité dans l'alimentation et la suppression des disparités en ce qui concerne la qualité et le prix de revient de l'eau.

La nécessité est donc apparue de mieux coordonner la production et la répartition des ressources en provenance d'un très grand nombre de points de prélèvement dispersés sur le territoire de la région, et même au-delà, afin de tendre vers un optimum économique dans l'exploitation des installations existantes et dans la réalisation des renforcements nécessaires à la satisfaction des besoins d'avenir. On espère ainsi mieux utiliser les fonds publics très importants consacrés à ce type de travaux.

L'étude est dirigée et financée, sous l'égide du District de la Région Parisienne, par un groupement réunissant:

- le District de la Région Parisienne déjà nommé;

- l'Agence Financière de Bassin Seine-Normandie;

- les principaux distributeur's d'eau publics ou privés de la Région.

L'étude est conduite de la façon suivante:

\section{Première partie}

- Evaluation, pour les horizons 1975-1980 et 1985, et suivant un certain découpage de la région, des besoins en eau dans chacune des zones ou unités de consommation d'eau.

- Construction et réglage d'un modèle mathématique purement technique, simulant le fonctionnement du groupement de réseaux actuel de la région.

- Exécution d'une première exploitation de ce modèle pour porter un jugement critique sur le fonctionnement actuel et orienter le choix des solutions d'avenit à examiner en deuxième partie.

\section{Deuxième partie}

- Utilisation des conclusions de l'étude des besoins en eau et d'un modèle complété pour tester, sur le plan technique, la cohérence d'un certain nombre de schémas d'avenir.

- Utillisation du même modèle pour étudier le niveau de sécurité offert par chaque schéma précédemment défini.

- Comparaison économique entre chacune des solntions proposées.

\section{Première partie}

\section{Ełude prospective des besoins en eau}

Les prévisions de consommation d'eau tiennent compte, d'une part des prévisions de population et d'emplois secondaires et tertiaires élaborées par les organismes de planification et de réflexion à long terme et, d'autre part, d'hypothèses d'évolution des consommations unitaires de la population et des emplois. 


\section{Zonage}

Le découpage géographique préalable en zones homogènes, du point de vue de la consommation, répond à la nécessité de prendre en compte les motivations locales et doit permettre la recherche des grandes liaisons à établir entre les sites de production et les lieux d'utilisation. Les villes nouvelles projetées sont, en particulier, traitées en zones indépendantes prédéterminées.

Les zones comprenant plusieurs communes sont définies à partir des critères suivants établis pour chacune de celles-ci :

- structure de la consommation actuelle et évolution depuis plusieurs années: importance relative des différents types de consommations (domestiques, des emplois) et consommations unitaires de ces types;

- facteurs socio-économiques qui caractérisent la consommation domestique (type d'urbanisation, équipement des logements, catégorie socio-professionnelle);

- programmes de développement jusqu'à l'horizon 1985 (population, emplois);

- configuration des systèmes de distribution existants, éventuellement.

Le classement des six cents communes en fonction de la structure de la consommation conduit à un zonage provisoire correspondant à quatre types. L'analyse typologique des facteurs socio-économiques, réalisée à l'aide d'un programme original, donne six types principaux suffisamment distincts et bien localisés. De la carte obtenue à l'aide de ces deux informations, un zonage final, comprenant sept zones dans Paris et trente-trois à l'extérieur, est mis au point, compte tenu de l'homogénéité du taux de croissance des communes provisoirement regroupées et de quelques ajustements liés aux linites des réseaux existants.

Chaque zone définie ci-dessus fait alors l'objet d'une étude particulière des consommations d'eau et des facteurs socio-économiques qui les expliquent.

\section{Etude des normes de consommation}

La connaissance plus précise du comportement des différents utilisateurs d'ean s'applique aux ménages, aux emplois secondaires et tertiaires. En fonction de certaines données connues pour l'avenir, les critères suivants sont recherchés pour l'évaluation des consommations domestiques unitaires :

- consommation et taille des communes: pas de lien;

- consommation et critères de niveau de vie et d'équipement du logement: les catégories socio-professionnelles, les mieux corrélées, sont retenues;

- consommation et critères de niveau de vie; corrélation multiple recherchée à l'ordinateur: $96,8 \%$ de la variance expliquée;

- consommation et population par type de commune: $95 \%$ de la variance expliquée.

Les consommations par emploi et branche d'activité sont tirées des statistiques disponibles et des résultats d'enquêtes complémentaires.

Les taux de croissance de ces différentes consommations unitaires sont ensuite recherchés sur la période 1962-1970.

\section{Prévisions de consommation d'eau}

Les consommations par zone, aux différentes étapes, sont calculées à partir des données définies dans les schémas de développement et des hypothèses relatives aux consommations unitaires. Ces dernières, découlant du type de la zone, sont établies suivant deux hypothèses: évolution linéaire et évolution exponentielle. Les prévisions de développement étant présentées sous forme de deux éventualités de croissance, la prévision de consommation d'eau comporte une hypothèse faible et une hypothèse forte.

Une étude parallèle des consommations par prélèvements directs en milieu naturel est réalisée en admettant que leur croissance sera proportionnelle à celle des consommations industrielles sur les réseaux. La prévision finale tient compte de la limite ou du devenir des ressources ainsi exploitées, et fait ressortir la production d'eau potable à envisager compte tenu du niveau prévisible des pertes et fuites.

\section{Etablissement ef calage du modèle mathématique}

Le modèle mathématique, représentant les systèmes actuels de distribution, est constitué d'après les cartes des réseaux en ne retenant, à partir des points d'alimentation ou de mise en charge, que les canalisations formant ossature à l'exclusion des sous-réseaux non directement concernés par les interconnexions envisageables. Le schéma comporte toutes les indications nécessaires à une simulation d'un fonctionnement en régime permanent: longueur, diamètre, nature, ancienneté des canalisations; dimensions des réservoirs, courbes caractéristiques des pompes; cotes des points caractéristiques.

Le calage du modèle est effectué d'après deux situations instantanées du réseau qui comporte initialement 1750 tronçons. Ces situations (une le matin et une l'après-midi du même jour) sont précisées par des campagnes de mesures se rapportant aux données suivantes:

- pressions sur $20 \%$ au plus des points de calcul;

- niveaux piézométriques dans les ouvrages de mise en charge (réservoirs, stations de pompage);

- débits à la sortie de ces ouvrages, en divers points des réseaux et au branchement de gros consommateurs.

Au cours des mesures effectuées de 5 en 5 minutes sur une période d'une demi-heure, l'exploitation est "figée 》 afin d'éviter l'apparition de tout phénomène transitoire.

La consommation d'eau totale mesurée est alors affectée à certains points du réseau en fonction de la répartition géographique des besoins, tirée des statistiques de facturation. Certains points de prélèvements représentent de gros consommateurs identifiés. Pour les autres, qui concernent des consommateurs de types variés, une pondération est effectuée compte tenu de l'heure des mesures et de l'importance relative des consommations moyennes domestiques et des emplois au lieu de prélèvement.

Le calage du modèle est ensuite réalisé à l'aide d'une suite de calculs all cours desauels les hypothèses sont modifiées jusqu'à obtention de valeurs voisines de celles mesurées. Des écarts maxima de quelques mètres d'eau en pression et de quelques pour cents en débit, sont obtenus après huit à douze essais par situation, suivant le processus ci-après : 
-- modification de la rugosité des tuyauteries, tant qu'elle demeure compatible avec le type ou la qualité des eaux et suffisamment homogène sur l'ensemble du réseau;

- introduction de partes de charge particulières représentant des singularités, des organes de réglage ou des ensembles complexes situés dans les ouvrages;

- correction d'indications erronées, décelées par le calcul et vérifiées in situ (altimétric, appareil de mesure, vanne de réglage, singularité);

- introduction de canalisations pour améliorer la représentativité du modèle;

- enfin, modification de la répartition de la consommation par transferts de débits pouvant représenter une part des fuites ou traduire une consommation anormale.

\section{Exploilation immédiate du modèle}

Le modèle calé est utilisé pour tester le fonctionnement actuel du réseau, tant en fonctionnement normal qu'en fonctionnement exceptionnel. Les essais correspondants se rapportent au fonctionnement en heure de pointe, ou consistent on une simulation du fonctionnement journalier réalisé par douze calculs automatiquement enchaînés, qui font ressortir en particulier l'état des réserves en fonction du mode d'explotitation adopté pour les ouvrages principaux.

Cette exploitation comprend les essais suivants:

- analyse du fonctionnement au débit de pointe de 1972;

- recherche de la consommation maximale possible, compte tenu des contraintes de pression et de production d'eau;

- simulations d'avaries (rupture de feeder, isolement d'une ou plusieurs alimentations, notamment au passage d'un nuage de pollution en rivière, etc.).

Ces différents tests permettent de porter un jugement sur les points forts ou faibles du système actuel, par l'examen de son aptitude à satisfaire les usagers (pressions résiduelles), de l'efficience des canalisations (pentes piézométriques et vitesses de l'eau), de l'évolution des réserves de régulation et de sécurité, enfin du niveau de sécurilé générale en cas d'incidents locaux. Ils déterminent également l'origine de l'eau consommée en chaque point du réseau.

\section{Deuxième partie}

L'objet de celte deuxiène phase d'étude est la détermination d'un schéma du réseau futur par la comparaison économique de plusieurs solutions prédéterminées. Ces solutions d'ensemble, on configurations, sont initialement définies en fonction des enseignements tirés de la première exploitation du modèle; elles doivent s'adapter, par une réalisation échelonnée adéquate, à la progression des besoins en eau et des ressources mobilisables jusqu'à l'année 1985. Représentées par le tracé des conduites et l'implantation des ouvrages généraux, elles font lobjet d'un premier dimensionnement a priori des divers éléments qui les composent, avant d'être mises au point par le calcul.

\section{Mise au point des différentes configurations}

La méthode générale consiste à vérifier, à l'aide du modèle complété et adapté, que chaque configuration est en mesure de satisfaire la progression des besoins de pointe. 11 est donc procédé à un premier ajustement des caractéristiques des ouvrages pour l'horizon 1985, puis à un choix des ouvrages nécessaires en 1975 et 1980 . Ces ajustements des configurations sont obtenus à l'issue de calculs de mise au point, réalisés à l'aide du modèle utilisé précédemment, complété par une évaluation systématique des investissements.

\section{Sous optimisation}

Une amélioration sur le plan économique peut être obtenue, après une mise au point plus ou moins fine des solutions apparaissant les plus intéressantes, par une adaptation plus stricte des diamètres des canalisations aux conditions de débit et de pression imposées, et par des réalisations mieux échelonnées dans le temps. Cette opération est effectuée à l'aide d'un programme de minimisation des diamètres des conduites à partir de débits de circulation précalculés par la méthode générale. Selon le degré de précision désirable on recherche:

- sous optimisation des diamètres : minimisation du réseali. de 1985 et définition des étapes 1975 et 1980 en fonction du schéma objectif ainsi déterminé;

- sous optimisation des diamètres et des échéances de réalisation: exploration de diverses solutions différentes par les caractéristiques des équipements et leur date de mise en service; quatre cheminements sont étudiés, le premier supposant que chaque équipement neuf est dimensionné définitivement pour 1985 à la date de sa mise en service, les autres correspondant à des objectifs successifs à plus court terme.

Quelle que soit la méthode, le niveau de sécurité offert par chaque solution et à chaque échéance est recherché par l'analyse des conséquences d'une défaillance de certains organes principaux sur les niveaux de pression.

\section{Bilan économique et conclusions}

Cette dernière opération se résume en un calcul du coût actualisé des dépenses afférentes à un certain nombre de solutions, effectué à l'aide d'un sous-programme du modèle.

Les données, prises en compte dans l'actualisation, sont le bordereau des prix, la période d'analyse, la durée de vie des équipements, le taux d'actualisation, les charges d'entretien et d'exploitation.

La conclusion de l'étude comporte une récapitulation de ce bilan économique, un avis surr le niveau de sécurité offert par chaque configuration, et divers autres éléments d'appréciation qui faciliteront le choix des organismes responsables de lalimentation en eau de la Région Parisienne. 


\section{Discussion}

Président : M. H. LORIFERNE

M. le Président remercie MM. Dargent et BANSARd pour :eur exposé fort documenté et poursuit :

M. Dargent a bien mis en humière les raisons pour lesquelles l'Agence Financière de Bassin, en liaison avec les distributeurs de la région parisienne, avait lancé ce modèle et quelles étaient les conclusions qu'elle espérait en tirer; M. BANSARD vous a expliqué les difficultés à vaincre poux l'établissement de ce réseau, en particulier les sujétions rencontrées pour son ca'age; celles-ci découlent du grand nombre de mailles que comportait ce dernier et des nombreux points de contrôle (pas toujours tous valables). Actuellement, ce modèle est établi et calé. Il reste à l'exploiter au mieux et ce n'est que dans que'ques temps que l'on pourra vous dire comment fonctionne le modèle et ce qu'on aura pu en tirer. Cela pourrait faire lobjet, vu son grand intérêt, d'un exposé - peut-être dans deux ans - quand on aura vraiment tiré de ce remarquable outil toute la substance.

Y-a-t-il des questions que vous désiriez poser, soit à $M$. Dargent soit à M. Bansard, demande M. le Président?

Quelles ont été les méthodes de mesure utilisées au cours de la campagne d'essais qui a permis de caler le modèle? demantle M. Rousset.
Nous avons procédé, répond M. BANSARd, à des mesures du niveau de l'eau dans les ouvrages, à des mesures de pression à l'aspiration et au refoulement des pompes et aussi sur les conduites du réseau,

Un problème commun aux deux modèles mathématiques dont on vient de parler, observe $M$. le Président, réside dans la sensibilité des résultats à la variation des "données d'entrée» fournie au modèle. A priori, seul le bon sens peut indiquer à quel niveau de «finesse » on doit s'arrêter pour obtenir une solution valable.

Il ne faut pas avoir, au départ, un nombre de données insuffisantes, mais un trop grand nombre augmente inutilement le cô̂t et la durée de l'étude pour un gain de sensibilité infime.

Dans ce domaine, estime M. BANSARD, il n'y a que des cas particuliers. Ainsi, dans une certaine zone, où seules deux conduites principales étaient modélisées, il a fallu représenter, en diamètres réels ou en diamètres fictifs, tout le réseau qui leur était connecté pour refrouver correctement l'équilibre des pressions entre les deux canalisations.

M. le Président clôt la discussion et donne la parole à MM. PoCHET et CHAUveT pour l'exposé de leur communication. 